\title{
Theoretical Virtues and Theorizing in Physics: Against the Instrumentalist View of Simplicity
}

\author{
Mousa Mohammadian (mmohamma@nd.edu)
}

ORCID: 0000-0002-2422-4979

This is a pre-print of a forthcoming article in Synthese Topical Collection "Simplicity out of Complexity? Physics and the Aims of Science."

\begin{abstract}
I argue that if (a) simplicity is a theoretical virtue and (b) some theoretical virtues are the constituents of the aims of theorizing in physics-i.e., theory choice and theory development in physics - and (c) scientific rationality is instrumental rationality, then simplicity cannot be a mere means to achieve the aims. I do this by showing that considering simplicity as a mere means brings about counterintuitive ramifications concerning scientific rationality. These counterintuitive ramifications can be avoided if we consider simplicity a constituent of the aims of theorizing in physics.
\end{abstract}

Keywords: simplicity, theoretical virtues, the aims of science, scientific rationality, instrumental rationality

Acknowledgment: I am grateful to Anjan Chakravertty, Janet Kourany, Faeze Fazeli, Oliver Traldi, and two anonymous referees for their helpful comments and suggestions.

\section{Introduction}

Scientific theories differ in a variety of ways. Some theories are about ordinary objects such as rocks, trees, and birds, while others are about extraordinary objects such as quarks and galaxies. String theory rarely, if at all, touches upon experiment and observation, while chemistry and primatology rely heavily on them. Despite all these differences, many scientists and philosophers of science hold that there are some characteristics that are good and desirable for all scientific theories including internal consistency, external consistency with well-established scientific theories, the empirical fit between theory and 
available data, accuracy, simplicity, explanatory power, non-ad hocness, predictive power, unification, and broad scope. These desiderata are generally known as "theoretical virtues" of scientific theories. ${ }^{1}$

Some philosophers divide theoretical virtues into two groups. Some of them are taken to be the constituents of the aims of scientific inquiry while others are simply means that should be used to achieve the aims. An example of this approach is proposed by Hempel (1979a, 1979b/2001). In his discussion about the aims of science, he begins with "an avowedly oversimplified construal” of science's aims: "science aims at establishing a sequence of increasingly comprehensive and accurate systems of empirical knowledge" (1979a, p. 51). Although it is already clear that Hempel understands the aims of science in terms of some theoretical virtues, he later moves towards a more complete construal of the aims of science by invoking "Kuhnian values"-i.e., consistency, simplicity, accuracy, fruitfulness, and scope (Kuhn 1977) — as well as some theoretical virtues that are not mentioned, at least explicitly, by Kuhn (e.g., predictive power):

It seems to me that most of these desiderata are best regarded as providing a fuller characterization of the goals of scientific theorizing rather than as instrumental norms aimed at enhancing the prospects of achieving those aims. (Hempel 1979a, p. 52) ${ }^{2}$

\footnotetext{
${ }^{1}$ Different catalogs of theoretical virtues can be found in the works of scientists and philosophers of science at least since Robert Boyle's "Notes on a Good and an Excellent Hypothesis" (1991, p. 119). Most of these catalogs have considerable overlaps. For some examples, see Hempel (1966, 1983), Quine and Ullian (1978), Kuhn (1977), Newton-Smith (1981), McMullin (1996, 2008), Lacey (1999), Lycan (1985), Douglas (2013), and Keas (2018).

${ }^{2}$ In a response to this paper, Kuhn (1983, p. 565) admits that Hempel's articulation of the aims of science in terms of theoretical virtues is an "improvement" over his own "puzzle-solving" account of the aim of science. Probably the most detailed account of the aims of science in terms of theoretical virtues can be found in Laudan (1984).
} 
However, not all theoretical virtues are among the constituents of the aims of science. Crucially, Hempel (1979a, p. 52) suggests that simplicity might be viewed as a means of achieving the aims of science rather than its constituent. In this regard, simplicity might not have an intrinsic value. Rather, it is scientifically valued when it leads us to those theoretical virtues that are constitutive of the aims of science. Hempel is not alone in proposing an instrumentalist view of simplicity. More recently, Sober (2015, p. 149) has endorsed this view too: "I am a reductionist about parsimony. If parsimony contributes to the achievement of some more fundamental epistemic aims, I am all for it. If it does not, I am not.”

In this paper, I argue against the instrumentalist view of simplicity. Although I present my argument against the background of Hempel's account, as we shall see, it goes beyond the specifics of his view regarding what theoretical virtues are considered as constituents of the aims of science or whether such theoretical virtues are truth-conducive or not. In general, I argue that if (i) simplicity is a theoretical virtue and (ii) some theoretical virtues are constituents of the aims of science and (iii) scientific rationality is instrumental rationality, then simplicity cannot be a mere means to achieve the aims. Rather, it is better to think of it as a constituent of the aims of science. Of the three parts of the argument's antecedent, I shall not discuss (i) because all the major catalogs of theoretical virtues that are discussed in the literature include simplicity. ${ }^{3}$ As we shall see, (iii) is also widely accepted among philosophers of science and (ii), I argue, is quite uncontroversial once, instead of science in general, we focus on theorizing in physics, which is in line with the subject-matter of this topical collection.

\footnotetext{
${ }^{3}$ For instance, simplicity is mentioned as a theoretical virtue in all the resources that are cited in footnote 1.
} 
The structure of the paper is as follows. Section 2 includes some preliminary remarks about theoretical virtues and 'the aims of science'. Section 3 argues that considering simplicity as a means for achieving the aims of science brings about serious counterintuitive results about the rationality of science.

\section{Preliminary Remarks}

In this section, first I discuss an important characteristic of theoretical virtues that plays a significant role in my argument. Then I use this discussion to explain what I mean by 'the aims of science' and provide a non-controversial version of (iii).

\subsection{Degrees of Theoretical Virtues}

Theoretical virtues can be exemplified in different degrees. Regarding external consistency, for instance, a theory might be consistent with more or less of the existing corpus of well-established scientific theories. A theory can achieve higher and higher degrees of explanatory power by suggesting more and more fundamental laws, causes, and mechanisms. Similarly, a theory can instantiate greater or lesser degrees of accuracy, simplicity, scope, and predictive power, both when it is compared with its rival theories or with its own earlier or later stages of development. Therefore, we can say that theory $T 1$ instantiates some theoretical virtues "better" than theory $T 2$ does when $T 1$ instantiates more of those theoretical virtues than $T 2$ does and/or it instantiates higher degrees of those theoretical virtues than $T 2$ does.

\subsection{The Aims of Science?}

Science is widely considered as an exemplar of rationality and many have strong intuitions indicating that a rational activity should have some aim(s). Yet, a persuasive account of the aims of science should 
deal with some major difficulties. For one thing, science is diverse in many aspects: it includes a diverse range of disciplines and practices that might be done for very different purposes by different practitioners of science. This makes it difficult, if not impossible, to come up with a particular formulation for "the aims of science" that can be applied to all disciplines, practices, and scientists. One might argue, for instance, that different research programs in evolutionary biology, string theory, clinical psychology, and metallurgy might be conducted for very different aims. A string theorist's aim for doing her research might be unification while a researcher at an industrial metallurgy lab might conduct an experiment, not for the sake of any particular epistemic goal but only to examine a practical utility of a metallic object. Such considerations have led some philosophers of science to argue that speaking of 'the aims of science' should be avoided altogether (Rowbottom 2014).

But for the purpose of this paper, fortunately, it is possible to make a maneuver around such difficulties by positing three provisions. First, since my discussion is structured around theoretical virtues, instead of science in general, I only focus on a particular, though essential, scientific practice, namely, scientific theorizing. Second, in accordance with the subject-matter of this topical collection, I content myself to theorizing in physics. So instead of saying that some theoretical virtues are constituents of the aims of science, I can simply say that some theoretical virtues are constituents of the aims of scientific theorizing in physics. Here, by 'theorizing', I mainly have two contexts in mind: the context of theory choice, that is, when physicists select between rival theories, and the context of theory development, when physicists work on an already selected theory to further develop it. Finally, these "aims of theorizing in physics" can be primarily understood in terms of those desiderata that are invoked by the scientific community (in this case, theoretical physicists) to appraise different cases of theory choice and 
theory development. In this sense, thus, when we say such-and-such are the constituents of the aims of physical theorizing, we are making a claim about the major standards of assessment of theory choice and theory development in the theoretical physics community. ${ }^{4}$ This claim is obviously empirical in the sense that the only reasonable way to find those desiderata is through studying the relevant practices of theoretical physicists-e.g., pedagogy, peer review, etc. However, it is quite uncontroversial that some of these desiderata are very well-known and very widely accepted by all the members of the community. ${ }^{5}$ For instance, I don't think anyone disagrees that a physical theory is considered to be better than its rival when, ceteris paribus, it instantiates higher degrees of external consistency, empirical adequacy, explanatory power, unification, and accuracy. Given these provisions, (iii) can be modified to a quite uncontroversial claim according to which some theoretical virtues are constituents of the aims of theory choice and theory development in physics (let's call them "aim-TVs"). That is, in selecting a physical theory as opposed to its rivals or in developing a theory to a more "advanced" stage, the aim is to attain a theory that instantiates more or higher degrees of aim-TVs.

\section{Simplicity and Scientific Rationality}

So of the three parts of my general argument's antecedent, it was shown that (i) and (ii) are quite uncontroversial. Similarly, (iii) is also widely accepted. Many philosophers-for example, Hempel (1979a), Newton-Smith (1981), Laudan (1984, Chapter 2), Giere (1989), Solomon (2001, Chapter 2), and Thagard (2004) — hold that scientific rationality is instrumental rationality, ${ }^{6}$ namely, it consists in

\footnotetext{
${ }^{4}$ Such approach, though not particularly about theoretical physics, is adopted by Kuhn (1977), McMullin (1982), Laudan (1984), and Longino (1990, Chapter 4).

${ }^{5}$ As Kuhn (1977) argues, this does not mean that different members of the community cannot have legitimate disagreements about the interpretation of theoretical virtues or their relative weight in theory choice.

${ }^{6}$ For a non-instrumental account of scientific rationality, see Friedman (2001, pp. 53-68).
} 
employing means that are justifiably believed to be conducive to achieving desired aims $(\mathrm{s}) .^{7}$ In his “Scientific Rationality: Analytic vs. Pragmatic Perspectives" (1979a), Hempel neatly articulates the requirements of instrumental rationality in the context of science:

in so far as a proposed methodological theory of science is to afford an account of scientific inquiry as a rational pursuit, it will have to specify certain aims of scientific inquiry as well as some methodological principles observed in their pursuit; finally, it will have to exhibit the instrumental rationality of the principles in relation to the goals. (1979a, p. 58)

Thus, a comprehensive account of rationality in science consists of three pillars: specifying the aims to be achieved, specifying the norms that should be followed or the means that should be used to achieve the aims, and establishing the instrumentality of norms or means for achieving the aims by showing that the aims can be achieved by following the norms or using the means). ${ }^{8}$

As mentioned before, according to Hempel, some theoretical virtues are constituents of the aims of science — namely, they are aim-TVs — while simplicity is a means of achieving the aims and hence it is not an aim-TV. Here, I argue that this instrumentalist view of simplicity cannot preserve the rationality

\footnotetext{
${ }^{7}$ Here, a distinction can be made between "subjective rationality" (or rationality vis-à-vis a scientist's beliefs) and "objective rationality" (or rationality vis-à-vis available evidence). A scientist's theory choice, for instance, might be subjectively rational because she believes that the chosen theory best instantiates the aim-TVs, but objectively irrational because her relevant beliefs are false-i.e., inconsistent with the evidence. In this paper, I avoid the problem of possible conflicts between objective vs. subjective rationality by assuming that scientists' relevant beliefs are always justified, namely, their beliefs are supported with the relevant facts of the matter. So, for instance, if scientists believe that $T 1$ is more accurate (or less explanatory powerful) that $T 2$, they are justified in their belief because their belief accord with the evidence. This, of course, does not mean that their beliefs are always true.

${ }^{8}$ As Rowbottom (2010, pp. 212-13) suggests, the achievability of the aims by following the norms or using the means can be also understood probabilistically: it is rational to adopt some means if it makes it more probable to achieve the aims. As we shall see, my arguments can also address probabilistic instrumentality. For the sake of brevity, in what follows, I primarily talk about "achieving the aims" rather than "making achieving the aims more probable."
} 
of science properly. For this reason, I focus on the third pillar of instrumental rationality to see if the instrumentality of simplicity for achieving aim-TVs is established.

In general, the instrumentality of a particular means for an aim might admit of four different kinds:

1. The means is necessary and sufficient for achieving the aims;

2. The means is necessary for achieving the aims;

3. The means is sufficient for achieving the aims;

4. The means is neither necessary nor sufficient for achieving the aims;

Consider, for instance, an impeachment trial of the president of the United States in the senate. If the aim is to remove the president from office, the concurrence of two-thirds of the senators is necessary and sufficient, the concurrence of at least half of them is necessary but not sufficient, the concurrence of three-fifths of the senators is sufficient but not necessary, and the concurrence of the senate minority leader is neither necessary nor sufficient.

Now, if simplicity is a means to achieve aim-TVs, what kind of means is it? It is obvious that simplicity is not sufficient (and hence necessary and sufficient) means for achieving aim-TVs. It is also quite clear that simplicity is not a necessary means to achieve aim-TVs. If it were, it would be impossible to achieve aim-TVs without gaining simplicity. But obviously, it is possible to add more (or more complicated) true laws or causal mechanisms to a theory and thereby to gain more unification, explanatory power, or predictive power while the theory loses some degree of simplicity.

That simplicity is not necessary for achieving other theoretical virtues is even true with respect to non-ad hocness, a theoretical virtue that is very closely related to simplicity. Obviously, adding an ad 
hoc modification to a theory-i.e., a modification whose sole purpose is to save a theory from refutation by explaining a particular difficulty without adding to the empirical content of the theory-makes the theory less simple. For this reason, Lacey $(1999,60)$ includes non-ad hocness under the umbrella of simplicity. Yet, resolving a theory's ad hocness does not necessarily make it simpler. Consider, for instance, the dark matter hypothesis introduced as an ad hoc hypothesis to explain the discrepancy observed between the results of measuring the distribution of mass in galaxies in two different waysnamely, first, by observing the orbital speeds of a galaxy's stars and calculating the masses through the physical laws of motion and, secondly, by adding up the masses of all the objects that can be seen in the galaxy. To resolve the ad hocness of this hypothesis, cosmologists have been trying to come up with a comprehensive cosmological theory in which the dark matter is neatly explained and empirically corroborated. The point is that it is possible for such a theory to be less simple than the current cosmological theory that includes the ad hoc dark matter hypothesis. In other words, the ad hocness of the theory might be resolved while the theory gets more complex. Therefore, simplicity is not even a necessary means to achieve non-ad hocness.

Thus, simplicity is neither a necessary nor a sufficient means to achieve aim-TVs. This, however, is not in itself problematic: a means can preserve its instrumentality for an aim without being a necessary and/or sufficient means for that aim. For instance, a means might make the achievement of an aim more probable or simply gets us closer to achieving the aim rather than getting us to the aim. Imagine, as an example, that Mehrnoosh is in New York and her aim is to travel to Chicago. Driving a car is neither necessary (because she can fly to Chicago) nor sufficient (because she can drive to Boston instead of Chicago) to achieve this aim. Yet, it can be a means of getting to Chicago from New York under certain 
conditions, such as careful attention to the directions of the roads, observing traffic rules, etc. But the mere existence of such conditions is not enough to make it rational for Mehrnoosh to drive a car for traveling from New York to Chicago. She should also be aware of those conditions and be able to satisfy them. Without such knowledge, even if she drives her car from New York and ends up in Chicago anyway, her action is not rational and her achievement is not a success but a mere lucky accident.

Similarly, if using simplicity as an instrument to achieve the aim-TVs is to be rational, two requirements should be satisfied. First, there must be certain conditions under which the instrumentality of simplicity for achieving aim-TVs is established. Second, it should be shown that physicists are aware of these conditions - that is, they have justified beliefs about what these conditions are-and apply them in their practice of physical theorizing. Only if there are such conditions and physicists are aware of them, utilizing simplicity to achieve aim-TVs is rational and, if successful, results in planned progress. But if there are no such conditions or, if there are, physicists are not aware of them, even if they take their chance on trying simplicity anyway, regardless of whether they succeed in achieving aim-TVs, their use of simplicity is not rational. If they succeed in achieving aim-TVs, it is a lucky accident. If they do not succeed, it is a failure. Anyway, it is not rational.

But it is not clear at all whether there really are specific conditions under which simplicity is instrumental for achieving other theoretical virtues. Hempel does not mention any such conditions. Interestingly, Sober (2015) who also endorses the instrumentalist view of simplicity—or, as he calls it, the reductionist view_admits that such conditions do not exist. Quite conversely, as Steel (2016) puts it, one of the major claims about simplicity that emerges from Sober's discussion is "contextualism" according to which such conditions depend on "defeasible, context dependent empirical assumptions" 
that change from one case to another. Moreover, if we hold that science as it is done now is, for the most part, rational, even a promising prospect for finding the conditions of the instrumentality of simplicity for achieving aim-TVs does not suffice to make pursuing simplicity rational. For finding such conditions tomorrow does not make today's appeal to simplicity rational.

There is, however, one way that we can make it rational to pursue simplicity as a means of acquiring other theoretical virtues without providing the conditions of instrumentality as discussed above. One might say that in doing science, we are like lost travelers in the forest. As Descartes (2006, p. 22) suggests, the rational thing for these travelers to do is to walk as straight as possible in one direction instead of wandering in circles or stopping in a place for some weak reasons. In doing science, one might suggest, invoking simplicity to achieve the aim-TVs is just like walking in a straight line when one is lost in a forest. Granted that there is no guarantee that the aims would be achieved, in absence of any other obvious course of action it is the rational thing to do. This proposal does make pursuing simplicity in physical theorizing rational but such "desperate" rationality seems too weak for someone who, like Hempel, demands a "conception of science as the exemplar of rationality" (1979a, p. 58).

But even if we come up with the conditions of the instrumentality of simplicity for other theoretical virtues - including those that are considered to be aim-TVs—-the instrumentalist view of simplicity brings about some counterintuitive results in the context of theory choice and the context of theory development. Here, I discuss four cases of such counterintuitive results and I argue that if we think of simplicity as an aim-TV, these counterintuitive results can be avoided.

Case One. Suppose there are two rival theories $T 1$ and $T 2$. Both theories exemplify similar degrees of aim-TVs and also have a similar prospect of achieving higher degrees of aim-TVs. The only 
difference is that $T 1$ is considerably simpler than $T 2$. Moreover, imagine that it is known that $T 1$ 's simplicity does not result in its instantiation of more (or higher degrees) of aim-TVs because the conditions of the instrumentality of simplicity are not satisfied. Now, according to the instrumentalist view of simplicity, the choice between $T 1$ and $T 2$ remains indifferent. After all, as much as the aim-TVs are concerned, the two theories are indistinguishable. Hence it is not irrational to adopt T2. I think, however, it is clear that in such cases physicists would adopt the simpler theory and take it to be the only rational choice. If simplicity is viewed as an aim-TV rather than a mere means to achieve other theoretical virtues, then the rational choice for physicists is to select the theory that, ceteris paribus, is the simplest theory.

Case Two. Imagine there are two rival theories T1 and T2. T1 has a slightly higher degree of a specific aim-TV - e.g., it is ever so slightly more accurate-than T2 but T2 is significantly simpler than T1. Otherwise, they are completely similar regarding their actual and prospective theoretical virtues. The instrumentalist view of simplicity entails that it is completely irrational to adopt $T 2$ because it is irrational to sacrifice the aims, namely, achieving higher degrees of aim-TVs, for the sake of a means, namely, simplicity. However, it seems quite intuitive that if $T 2$ 's relative degree of simplicity is significant enough while T1's relative degree of accuracy, say, is trivial enough, it is, to say the least, not irrational to adopt the simpler theory (i.e., T2). Again, once we view simplicity as an aim-TV, then we can make a good sense of this intuition: since simplicity is also an aim—rather than a mere means-a physicist is rational in selecting a greatly simpler but slightly less accurate theory because such a theory might instantiate a higher overall degree of all aim-TVs. 
Case Three: As a result of a scientist's works on a theory, the theory achieves a great deal of simplicity while it loses an insignificant degree of an aim-TV, e.g., an insignificant degree of accuracy or scope. According to the instrumentalist view of simplicity, no matter how significantly the theory gets simpler and how insignificantly it gets less accurate, what the scientist has done is irrational and her work is regressive rather than progressive. For she has compromised the aims of science, namely, an aim-TV, for the sake of a mere means. This, again, seems quite counterintuitive. If the achieved degree of simplicity is great enough and the lost degree of accuracy is insignificant enough, it seems reasonable to say that the scientist's work constitutes some progress and it is rational. This also shows that simplicity is better to be viewed as an aim-TV rather than a mere means to achieve other theoretical virtues.

Cases Four: Imagine that there is a real prospect for a theory to achieve a significant degree of simplicity without any change in its aim-TVs. For example, there is a real prospect of making a theory simpler through some mere notational changes without any change in its aim-TVs. According to the instrumentalist account of simplicity, it is not rational for a scientist who is aware of this prospect to try to achieve a higher degree of simplicity for the theory when she knows that it does not result in attaining any aim-TVs. (Going back to the example of driving a car, it is irrational to drive a car with the intention of getting to a specific destination when we know in advance that it does not result in reaching or getting closer to that destination.) But it seems obvious that under such conditions, namely, when there is no concern for losing aim-TVs, simplicity is rationally desired even if it does not result in attaining more (or higher degrees of) aim-TVs. In fact, it might be even argued that if scientists do not make any attempt to make the theory significantly simpler when they are aware that there is a real prospect for it, they are acting irrationally. Once again, this intuition suggests that simplicity is not a mere means to achieve 
some aim-TVs. Rather, even if making a theory simpler does not result in achieving any of the perceived aim-TVs, it is valuable in its own right. Thus, simplicity is better to be regarded as an aim-TV rather than a mere means.

\section{Conclusion}

If my arguments are sound, I have shown that if simplicity is a theoretical virtue and some theoretical virtues are the constituents of the aims of physical theorizing, then considering simplicity as a mere means to achieve aim-TVs results in problems and counterintuitive cases concerning scientific rationality understood in terms of instrumental rationality. These problems are resolved once we reject the instrumentalist view of simplicity and consider it as an aim-TV. 


\section{References}

Boyle, R. (1991). Selected philosophical papers of Robert Boyle. (M. A. Stewart, Ed.). Indianapolis: Hackett Publishing Company.

Descartes, R. (2006). A discourse on the method of correctly conducting one's reason and seeking truth in the sciences. (I. Maclean, Trans.). Oxford: Oxford University Press.

Douglas, H. (2013). The Value of Cognitive Values. Philosophy of Science, 80(5), 796-806.

Friedman, M. (2001). Dynamics of Reason. Stanford: CSLI Publications.

Giere, R. N. (1989). Scientific rationality as instrumental rationality. Studies in History and Philosophy of Science Part A, 20(3), 377-384.

Hempel, C. G. (1966). Philosophy of natural science. Upper Saddle River, NJ: Prentice-Hall.

Hempel, C. G. (1979a). Scientific Rationality: Analytic vs. Pragmatic Perspectives. In T. S. Geraets (Ed.), Rationality To-Day/La Rationalite Ajiourd'hui (pp. 46-58). Ottawa: The University of Ottawa Press.

Hempel, C. G. (1979b/2001). Scientific Rationality: Normative versus Descriptive Construals. In J. H. Fetzer (Ed.), The Philosophy of Carl G. Hempel: Studies in Science, Explanation, and Rationality (pp. 357-71). New York: Oxford University Press.

Hempel, C. G. (1983). Valuation and Objectivity in Science. In R. S. Cohen \& L. Laudan (Eds.), Physics, Philosophy and Psychoanalysis (pp. 73-100). Dordrecht: Springer.

Keas, M. N. (2018). Systematizing the theoretical virtues. Synthese, 195(6), 2761-2793.

Kuhn, T. S. (1977). Objectivity, Value Judgment, and Theory Choice. In The essential tension: selected studies in scientific tradition and change (pp. 320-339). Chicago: University of Chicago Press.

Kuhn, T. S. (1983). Rationality and Theory Choice. The Journal of Philosophy, 80(10), 563-570.

Lacey, H. (1999). Is science value free?: values and scientific understanding. New York: Routledge.

Laudan, L. (1984). Science and values: the aims of science and their role in scientific debate. Berkeley: University of California Press.

Longino, H. E. (1990). Science as social knowledge: values and objectivity in scientific inquiry. Princeton, N.J.: Princeton University Press.

Lycan, W. G. (1985). Epistemic value. Synthese, 64(2), 137-164.

McMullin, E. (1982). Values in Science. PSA: Proceedings of the Biennial Meeting of the Philosophy of Science Association, 1982, 3-28.

McMullin, E. (1996). Epistemic Virtue and Theory Appraisal. In I. Douven \& L. Horsten (Eds.), Realism in the Sciences (pp. 13-34). Leuven, Belgium: Leuven University Press.

McMullin, E. (2008). The Virtues of a Good Theory. In S. Psillos \& M. Curd (Eds.), The Routledge Companion to Philosophy of Science (pp. 498-508). New York: Routledge.

Newton-Smith, W. H. (1981). The Rationality of Science. London: Routledge.

Quine, W. V., \& Ullian, J. S. (1978). The web of belief (2nd ed.). New York: McGraw-Hill. 
Rowbottom, D. P. (2010). Evolutionary Epistemology and the Aim of Science. Australasian Journal of Philosophy, 88(2), 209-225.

Rowbottom, D. P. (2014). Aimless science. Synthese, 191(6), 1211-1221.

Sober, E. (2015). Ockham's razors: a user's manual. Cambridge: Cambridge University Press.

Solomon, M. (2001). Social empiricism. Cambridge, MA: The MIT Press.

Steel, D. (2016). Ockham's Razors: A User's Manual. Notre Dame Philosophical Reviews. https://ndpr.nd.edu/news/ockhams-razors-a-users-manual/.

Thagard, P. (2004). Rationality and Science. In A. R. Mele \& P. Rawling (Eds.), The Oxford Handbook of Rationality (pp. 363-379). New York: Oxford University Press. 